\title{
High Temperature Increased Lignin Contents of Poplar (Populus spp) Stem via Inducing the Synthesis Caffeate and Coniferaldehyde
}

\section{Xianyan Zhao}

Qilu University of Technology

\section{Panpan Li}

Qilu University of Technology

\section{Xingwang Liu}

Qilu University of Technology

\section{Tianyu Xu}

Qilu University of Technology

\section{Yuqing Zhang}

Qilu University of Technology

\section{Haifeng Meng}

Tai'an Forestry Protection and Development Center

\section{TaoXia ( $\nabla$ txia@qlu.edu.cn )}

Qilu University of Technology

\section{Research Article}

Keywords: Lignin, High temperature, Lignin metabolic intermediates, PtrMYBs, PtrC3'H1, PtrCCR2, Transcript and metabolic profiling

Posted Date: September 17th, 2021

DOl: https://doi.org/10.21203/rs.3.rs-798875/v1

License: (a) (1) This work is licensed under a Creative Commons Attribution 4.0 International License. Read Full License 


\section{Abstract \\ Background}

Lignin contributes to plant resistance to biotic and abiotic stresses and is dominantly regulated by enzymes which catalyze the generation of metabolites intermediates in lignin synthesis. However, the response of lignin and its key regulatory factors to high temperature stress are poorly unknown.

\section{Results}

Here, this finding revealed that the content of lignin in poplar (Populus spp) stem increased after three days of high temperature stress treatment. In fourteen metabolic intermediates of lignin biosynthetic pathway with targeted metabolomics analysis, caffeate and coniferaldehyde increased evidently upon heat stress. C3'H ( $p$-Coumaroylshikimate 3-hydroxylase) and CCR (Cinnamoyl-CoA reductase) are recognized to catalyze the formation of caffeate and coniferaldehyde, respectively. Transcriptome data and RT-qPCR (reverse transcription-quantitative real-time polymerase chain reaction) analysis found the high transcriptional level of PtrMYBs (PtrMYB021, PtrMYB074, PtrMYB85, PtrMYB46), PtrC3'H1 (Potri.006G033300) and PtrCCR2 (Potri.003G181400), suggesting that they played the vital role in the increase of lignin and its metabolic intermediates induced by high temperature.

\section{Conclusions}

The discovery of key regulators and metabolic intermediates in lignin pathway that respond to high temperature provides a theoretical basis for quality improvement of lignin and the application of forest resources.

\section{Background}

Forest resources not only have great application value for vegetation restoration, soil erosion prevention and saline-alkali land restoration, but also can be used for the development of biomass and fiber energy [1]. Lignin plays a vital role in cell wall formation, especially in wood and bark, and it permits the xylem to maintain a certain amount of water transport, mechanical support, growth and development for plants [2]. However, lignin is also a major obstacle to the utilization of forest resources [3, 4]. Therefore, the study of lignin is of great significance to the utilization of forest resources. Lignin synthesis is a complex network, including the lignin monomers biosynthesis, transport and polymerization process [5]. Lignin monomers are synthesized in the cytoplasm through a series of reactions including hydroxylation, deamination, reduction, methylation and transportation to the apoplast [5]. Seconldly, lignin is produced by the polymerization of lignin monomers in the secondary cell wall [5-8]. According to the differences in lignin monomers and crosslinking methods, lignin monomers can be classified as three mainly form, including p-hydroxyphenyl $(\mathrm{H})$, guaiacyl $(\mathrm{G})$, and syringyl $(\mathrm{S})[5,8]$. In the formation of lignin monomer, 
many enzymes are involved in its formation, and the expression level of the genes encoding these enzymes directly affects the content and deposition of lignin [5, 8]. Lignin is polymerized from the monomers of phenylpropane derivatives. Recently, researches showed that the change of phenylpropanoid biosynthetic enzymes in transgenic poplar has great influence on lignin content [9]. Phenylalanine ammonia-lyase (PAL), cinnamate 4-hydroxylase $(\mathrm{C} 4 \mathrm{H})$, and 4-coumarate CoA ligase (4CL) are three enzymes which are related with the first three catalytic reactions of the traditional phenylpropane pathway, respectively [8-11]. The decline of PAL, C4H and 4CL expression reduced the lignin content [8-11]. Many other enzymes such as quinate/shikimate p-hydroxycinnamoyltransferase (HCT), p-coumaroylshikimate 3-hydroxylase (C3'H), caffeoyl shikimate esterase (CSE), caffeic acid Omethyltransferase (COMT), and caffeoyl-CoA 0-methyltransferase (CCOAOMT), cinnamoyl-CoA reductase (CCR), ferulate 5-hydroxylase (F5H), cinnamyl alcohol dehydrogenase (CAD), are essential to maintain a normal level of lignin content $[12,13]$. The absence of any enzyme more or less affects the synthesis of lignin $[12,13]$.

$\mathrm{C}^{\prime} \mathrm{H}$ is a phenolic enzyme that catalyzes the production of p-coumaric acid to caffeic acid and pcoumaryl shikimic acid to catechylshikimic acid. The expression level of $C 3^{\prime} H$ directly affects the lignin content and $\mathrm{S} / \mathrm{G}$ ratio $[14,15]$. CYP98A3 gene was isolated in early bioinformatics experiments, which may encode C3'H enzyme protein in Arabidopsis [16]. In alfalfa, the content of lignin in transgenic plants with increased $C 3^{\prime} H$ expression was $5 \%$ higher than that in wild-type plants $[17,18]$. The lignin content in $P$. alba $\times$ P. grandidentata reduced significantly when the expression of $C 3^{\prime} H$ was inhibited $[14,19]$. Although the reduction of $C 3^{\prime} H$ expression will lead to dwarfed phenotype, relevant studies have shown that the specific components in mediator complex can contribute to the normal growth of plants and the increase of $\mathrm{H}$-type lignin, which is conducive to the conversion of bioenergy [20].

$C C R$ catalyzes the generation of hydroxycinnamaldehydes from hydroxycinnamoyl-CoA esters and its down-regulation cause the decline of lignin content [21, 22]. CCR1 mutant in maize changed the lignin deposition in the walls of the sclerenchymatic fibre cells surrounding the vascular bundles and lignin content [23]. Besides, reduction of lignin caused by decreased CCR expression brings about lower ferulic acid resulting in a decrease in polymers $[24,25]$. Down-regulation of $C C R$ gene expression can reduce the conversion of poplar into ethanol and even the biomass of poplar [22]. A recent study found that CCR2 knockdown can reduce lignin content in poplar trees without affecting their normal growth [26]. Besides, the overexpression of OsCCR can increase the lignin content and enhance the resistance to pathogenic bacteria in rice [27].

COMT is the methylation enzyme of lignin biosynthesis, which is responsible for the methylation of lignin precursor [28]. It catalyzes the methylation of a number of substances such as 5-hydroxy coniferyl alcohols, caffeoyl, free acids and aldehydes [28]. Inhibition of COMT transcription level in tobacco and poplar decreased lignin content $[29,30]$. In ryegrass, the biomass and phenotype unchanged with COMT1 expression disturbed [31]. Significantly, the economic value of ryegrass was increased by the reduction of lignin and its components [31]. The transcriptional level and enzyme activity of COMT can directly or indirectly affect the production of lignin, coumarin, flavonoids, organic acids and other metabolites [32]. 
Therefore, COMT may play a key role in promoting the growth and development of plant and its adaptation to the environment, as well as in the synthesis of ferulic acid.

NAC and MYB transcription factors are the two most important classes of transcription factors that regulate lignin [33]. NAC transcription factors PtrVND6 and PtrSND1 regulate lignin synthesis involved in poplar growth and development [34]. Downstream of NAC transcription factors, MYB transcription factors such as AtMYB46 and AtMYB83 are involved in lignin synthesis by regulating the expression of some structural genes in the lignin synthesis pathway [33]. Thus, it is crucial to explore the involvement of environmental factors in lignin synthesis through NAC and MYB transcription factors.

Poplar (Populus spp), which is an important forest resource, is served as a model material to study the basic biological characters of trees except for shelter forest, road greening and papermaking [35]. With the intensification of human activities, the occurrence of extreme weather is increasingly frequent. Therefore, it is of great significance for the utilization of bioenergy to understand the molecular mechanism of lignin response to extreme weather such as high temperature and explore candidate genes related to lignin synthesis and metabolism in response to high temperature stress in poplar.

\section{Results}

\section{Changes of lignin content in poplar stem caused by high temperature}

The biosynthesis of lignin can be altered by kinds of abiotic and biotic stresses [4,36]. To investigate the effect of high temperature on lignin content of poplar, poplar " $84 \mathrm{k}$ " of 40 days old tissue culture plantlets were treated with $35^{\circ} \mathrm{C}$ high temperature and $25^{\circ} \mathrm{C}$ normal temperature. Compared to the plantlets grown at normal temperature of $25^{\circ} \mathrm{C}$, determination of lignin content showed a slight increase in lignin content when exposed to high temperatures (Fig. 1). The results showed that poplar tissue culture plantlets tended to accumulate more lignin to resist heat stress when exposed to high temperature.

\section{Effect of high temperature treatment on metabolites in lignin synthesis pathway}

In order to further explore the substance in lignin synthesis pathway, targeted metabolomics analysis of 14 metabolites in the lignin synthesis pathway was performed. Lignin metabolism intermediates such as sinapic acid, coniferyl alcohol, p-coumaric acid, sinapyl alcohol, p-coumaryl alcohol, caffeyl alcohol, caffeyl aldehyde were detected but could not be quantified and analyzed because of their low levels. Among the seven intermediates of lignin metabolism quantified,sinapinaldehyde, p-coumaraldehyde, Iphenylalanine, ferulic acid and cinnamic acide showed no significant changes at different time periods after high temperature treatment (Supplementary materials 1; Fig. 2). However, it was found that caffeate and coniferous aldehyde always showed an upward trend in different periods after high temperature 
treatment, which may be the main reason for the increase of lignin content in poplar under high temperature stress (Supplementary materials 1; Fig. 2).

\section{Trait of transcriptional database libraries}

Both transcription factors and structural genes are involved in lignin synthesis by affecting the relevant enzymes in the lignin pathway. In order to investigate which genes are involved in the regulation of lignin after high temperature stress, $18 \mathrm{cDNA}$ libraries (6 samples, 3 replicates) from $35^{\circ} \mathrm{C}$ treated poplar stems at different time periods were established. 892458006 high-quality reads were obtained and transcriptome data were stored in NCBI database with SRA accession number PRJNA742770. About 70.69-74.14\% reads were matched to the genome. It was found that the percentage (about 68.25$71.80 \%$ ) of unique reads and clean reads in the genome was not much different. Therefore, the data quality and quantity of the transcriptome were reliable (Supplementary materials 2).

\section{Characterization of differential genes expression profiles of poplar stem in response to high temperature}

Five two-by-two comparisons, including 0 hour versus 6, 12, 24, 48, 72 hour respectively, were used to analyze the expression profiles of differential genes in the response to high temperature treatment in poplar stems. By correlating FPKM values, the FPKM heat map was constructed. The correlation value of three repeat samples at the same time was 1 while the correlation values of heat maps at normal and cold temperature at different period were all less than 0.8 , indicating a high degree of consistency in biological repetition with high temperature treatment (Fig. 3A).

The genes with log2 foldchange $\geq 1$ and FDR $\leq 0.05$ were significant DEGs. According to the analysis of transcriptome data, there were $6480,2421,1640,1452,778$ genes changed at 6, 12, 24, 48, and 72 hours, respectively (Supplementary materials 3; Fig. 3B). In detail, compared with 0 hour, the transcription level of $3274,1446,939,852,534$ genes increased respectively, while the transcription level of $3206,975,703$, 600,244 genes decreased after 6, 12, 24, 48, and 72 hours high temperature treatment (Supplementary materials 3; Fig. 3B). The studies showed that high temperature can stimulate the expression of related genes in poplar stem, and the gene change caused by high temperature reduced with time.

\section{Functional analysis of differential expressed genes (DEG)}

To further study the comprehensive annotation of DEGs in response to heat stress, the COG, KOG, GO and KEGG, protein databases were applied to annotate all the DEGs.

Twenty-six functional clusters were used for COG analysis. The COG analysis varies with the processing time. In general, both COG and KOG analysis revealed that the differential genes were mainly involved in inorganic ion transport and metabolism, carbohydrate transport and metabolism, energy production and conversion, signal transduction mechanisms, RNA processing and modification, posttranslational modification, protein turnover, chaperones, general function prediction only, cell wall/membrane/envelope biogenesis and other pathways (Supplementary materials 4, Supplementary materials 5). As can be seen 
from the results, the pathways involved in the protein data analysis of KOG and COG are almost identical. In addition, the changes of genes related to secondary metabolites biosynthesis, transport and catabolism suggest that some secondary metabolites may also participate in the response of poplar stems to high temperature. This indicated that poplar responds to the adverse effects of high temperature in a variety of ways, including energy conversion and signal transduction. For GO enrichment analysis, three terms including molecular function, biological process and cellular component were used to classify the DEGs function. Among all DEGs, 3319 genes were compared to the $\mathrm{GO}$ enrichment data. Of which, $1610,665,466,423$, and 228 genes changed after high temperature treatment of $6,12,24,48$, and 72 hours, respectively. The changed genes were mainly related to cell, organelle, cell part, cell junction, and membrane in cellular component; catalytic activity and binding in molecular function; and metabolic process, cellular process, single-organism process, response to stimulus, biological regulation in biological process (Supplementary materials 6). By analyzing the biological process, it was found that genes related toplant-type secondary cell wall biogenesis, the lignin catabolic process and lignin biosynthetic process which may influence lignin content changed significantly (Fig. 4).

Differential genes were selected for annotation analysis of KEGG database after high temperature treatment at $6,12,24,48$ and 72 hours. The results showed that the differential gene enrichment pathway decreased with the extension of treatment time (Supplementary materials 7). KEGG enrichment analysis at $0 \mathrm{~h}$ vs $6,12,24,48,72 \mathrm{~h}$ demonstrated that the enrichment pathways were mainly involved in starch and sucrose metabolism, biosynthesis of amino acids, plant hormone signal transduction, carbon metabolism, phenylpropanoid biosynthesis, etc (Supplementary materials 7). In general, the KEGG enrichment analysis of phenylpropanoid biosynthesis may be related with lignin synthesis.

\section{Lignin synthesis related genes responding to high temperature stress}

To investigate the genes involved in lignin synthesis in response to high temperatures, MYB, NAC and some structural genes in lignin synthesis pathway were analyzed. The results showed that a total of 843 genes may be involved in the process of lignin synthesis in poplar in response to high temperatures. In detail, there were 9 NACs, 558 MYBs, 6 PALs, 1 C4H, 2 C3'Hs, 16 4CLs, 35 HCTs, 32 CCRs, 1 COMT, 3 F5Hs, 4 CCOAOMTs, 42 CADs, 46 LACs, and 88 PODs (Supplementary materials 8).

The trends of the above lignin-related genes were analyzed by RPKM values (Fig. 5). Among the exchanged gene in lignin synthesis, PtrC3'H1 (Potri.006G033300), PtrCCR2 (Potri.003G181400), PtrMYB021 (Potri.009G053900) and PtrMYB074 (Potri.015G082700), PtrMYB103/46 (Potri.003G132000), PtrMYB090 (Potri.015G033600), PtrMYB161 (Potri.007G134500), PtrMYB3 (Potri.001G267300), PtrMYB125/85 (Potri.003G114100), PtrMYB093 (Potri.004G138000) may be involved in high temperature-induced synthesis of caffeate, coniferaldehyde and lignin. The other genes with more significant changes might also be involved in the process of lignin synthesis caused by high temperature.

\section{RT-qPCR analysis of major lignin synthesis related genes}


According to the network map of lignin synthesis pathway, some transcription factors such as MYBs, NACs and structural genes were found to be involved in the synthesis of caffeate, coniferaldehyde and lignin in response to high temperatures in poplar. By searching for related genes and designing primers, the transcript levels of several important genes, PtrC3'H1 (Potri.006G033300), PtrCCR2 (Potri.003G181400), PtrMYB021 (Potri.009G053900) and PtrMYB074 (Potri.015G082700), PtrMYB103/46 (Potri.003G132000), PtrMYB090 (Potri.015G033600), PtrMYB161 (Potri.007G134500), PtrMYB3 (Potri.001G267300), PtrMYB125/85 (Potri.003G114100), PtrMYB093 (Potri.004G138000), were examined by RT-qPCR analysis. The results showed that the transcript levels of these genes and the data from transcriptome analysis were consistent. It indicated that the transcriptome data were reliable and reproducible, and that these genes may play important roles in the regulation of lignin synthesis in response to high temperature in poplar seedlings.

\section{Discussion}

\section{The lignin content increased under high temperature stress}

High temperature stress is one of the major environmental factors that affect plant distribution and development and limit plant resource utilization [37]. Because of a series of advantages such as small genome, rapid growth and high economic value, poplar is a model for studying forest plants and also suitable for transcriptome study [35]. In this study, poplar tissue culture plantlets "84K" was used as the research material, and treated at $35^{\circ} \mathrm{C}$ for $6,12,24,48$, and 72 hours, it was found that the lignin content in the stem of poplar tissue culture seedlings increased significantly after high temperature treatment (Fig. 1). Lignin, a class of phenylpropane polymers, is essential for plant growth and development and in response to biotic and abiotic stress stimuli [38]. Once the stem of a plant is exposed to high temperatures, the cell walls rapidly lignify [39]. Lignin accumulates and is deposited in the cell wall, strengthening the stiffness of the cell wall, making the xylem cell wall less permeable to water and which facilitates the long-distance transport of water, minerals and organic matter in plants [40]. When plants are subjected to high temperature stress, they will enhance their resistance to stress by synthesizing more lignin.

The caffeate and coniferaldehyde may be the key factor for the increase of lignin content of poplar stem responding to high temperature

Lignin synthesis involves the production of many intermediate metabolites $[13,18,41]$. It was found that intermediates and their secondary metabolites produced by phenylpropanoid metabolic pathway not only improved disease resistance but also regulated and promoted plant resistance to abiotic adversities such as low temperature, high temperature, and UV radiation [42]. Under abiotic stresses such as ozone and drought, the content of phenylpropanoid metabolic secondary organisms including gallic acid, caffeic acid, and coumaric acid increased [42, 43]. Similarly, our study found that high temperature promoted the accumulation of caffeic acid. Caffeic acid is a key intermediate in the lignin metabolic pathway and is ubiquitous in the plant kingdom [44, 45]. In addition, mechanical injury, ethylene and methyl jasmonate 
treatment caused changes of caffeic acid [45]. The increase of caffeic acid content caused an increase of lignin content [46]. Therefore, it is possible that the elevation of caffeic acid stressed by high temperature is the cause of lignin elevation.

In addition to the elevated caffeic acid content, the coniferaldehyde content also increased after high temperature treatment. Coniferaldehyde is a key intermediate metabolite in lignin synthesis and sometimes functions as a lignin monomer to influence lignin synthesis [47, 48]. Coniferaldehyde also modulates the lignin metabolic pathway by altering the content and composition of lignin, including through the conversion to ferulic acid and its derivatives [49]. Coniferaldehyde content of lignin and other components filled in the cell wall can effectively inhibit the degradation of the maize cell wall [50]. In addition, physical, biochemical and mechanical properties of lignin altered because of the amount of coniferaldehyde residues $[3,9,51,52)$. In a word, the synthesis of these substances may be an important reason for the elevated lignin content caused by high temperature.

\section{The possible genes involved in lignin synthesis of poplar stem responding to high temperature stress}

The synthesis of caffeic acid is regulated by several genes. P-coumarate to caffeate reactions are mainly catalyzed by C3'H [53]. C3'H belongs to cytochrome P450 class. Schoch et al. cloned and isolated the C3'H gene from cytochrome P450 of Arabidopsis genome [16]. Numerous studies have shown that C3'H is the rate-limiting enzyme of phenylpropanoid pathway in the lignin biosynthetic pathway, which can catalyze the $\mathrm{C} 3$ hydroxylation reaction on the benzene ring of the phenylpropanoid structure and can determine the carbon source flow of lignin monomers. Down-regulation of $C 3^{\prime} H$ expression can both reduce lignin content and change monomer composition, which can reduce the cost of plant papermaking [54]. In hybrid poplar (Populus grandidentataalba), lignin monomer composition and lignin content were significantly reduced in $C 3^{\prime} H$ RNAi strains [14]. Among the two $C 3^{\prime} H$ subjected to high temperature-stimulated expression changes in this study, PtrC3'H1 (Potri.006G033300) had a very high similarity with $C 3^{\prime} H$ (GenBank accession no. EU391631) and its transcript levels were significantly elevated by high temperature stress. This suggested that PtrC3'H1 (Potri.006G033300) may be involved in caffeic acid production and thus affect lignin content after high temperature induction.

There are two directions regarding the destination of caffeic acid, one is the synthesis of ferulic acid in the presence of COMT (caffeic acid O-methyltransferase); the other is the generation of caffeoyl-CoA in the presence of 4CL (4-coumarate:CoA ligase) [53]. In hybrid poplar (Populus trichocarpa and Populus deltoides), overexpression of Pto4CL gene was effective in increasing lignin content [55]. The Pt4CL 1 gene was specifically expressed in organs with high lignin content such as xylem and was involved in the lignin synthesis process in aspen [56,57]. Among the 16 high temperature-induced $4 C L s$, the transcript expression levels of two genes, (Potri.004G102000) and (Potri.017G112800), were consistently reduced by high temperature stimulation, suggesting that the reduction in their transcript levels may have influenced the elevation of caffeic acid. In this study, it was found that only one PtrCOMT 
(Potri.006G120000) was induced by high temperature and its transcript level was reduced only at $6 \mathrm{~h}$. This may also be one of the reasons for the elevated caffeic acid content.

Coniferaldehyde synthesis is influenced by several genes. Among them, CCR and COMT have the ability to promote the production of coniferaldehyde, while F5H and CAD make coniferaldehyde to produce other substances. It was found that $32 \mathrm{CCRs}, 1 \mathrm{COMT}, 3 \mathrm{~F} 5 \mathrm{Hs}$ and $42 \mathrm{CADs}$ were induced by high temperature in this study. Among them, PtrCOMT (Potri.006G120000), whose expression was reduced by high temperature induction, was not responsible for the elevation of coniferaldehyde. Lignin contents in downregulated transgenic strains of CCR1 gene were significantly lower compared to wild type [22]. 12 of 32 CCRs induced by high temperature significantly elevated genes may be involved in high temperatureinduced coniferaldehyde and lignin synthesis. PtrCCR2 (Potri.003G181400), which was related with lignin content, exhibited evidently high expression after heat induction [26]. Besides, two genes (Potri.005G117500, Potri.007G016400) were up-regulated by high temperature induction in three $\mathrm{F} 5 \mathrm{Hs}$, indicating that they are not factors in coniferaldehyde elevation. However, there was no significant reduction in PtrCADs transcription levels among the numerous heat-induced PtrCADs, suggesting that PtrCADs may be not responsible for the elevation of coniferaldehyde.

\section{Analysis of transcription factors that may affect lignin synthesis in response to high temperatures}

Lignin synthesis is also regulated by transcription factors. The NAC MYB-based gene regulatory network (NAC-MYB-GRN) is widely considered to be the main pathway regulating lignin synthesis [33]. NAC is the primary network controlling lignin synthesis [33]. Downstream of NAC transcription factors are some MYB transcription factors such as AtMYB46 and AtMYB83 which is thesecondary regulatory network [33]. NAC and MYB affect lignin synthesis by regulating the transcription levels of some structural genes that are critical in the process of lignin synthesis.

NAC transcription factors, especially VND6 and SND1, are thought to be the master regulators of SCWs $[34,58]$. In poplar, PtrVND6 and PtrSND1 can participate in the synthesis of lignin as well as cellulose, hemicelluloses by affecting the differentiation of secondary cell walls (SCWs) [34]. Overexpression of VND6 and SND1 caused impaired lignin content and unhealthy plant development [34]. However, among the nine NACs identified that were induced by high temperature in this study, the changes were not obvious, and none of them were reported to be involved in lignin synthesis or SCWs formation. Some MYB transcription factors acted as the downstream of NAC transcription factors and are involved in lignin synthesis by directly binding to the promoters of lignin structural genes [4]. PtrMYB021 and PtrMYB074 regulated the growth and development of poplar by participating in lignin production [59]. In addition, several other MYB transcription factors were found to be involved in the lignin metabolic pathway [59]. By analyzing 558 MYB transcription factors induced by high temperature, it was found that the transcript levels of PtrMYB021 and PtrMYB074 were significantly elevated by high temperature induction. MYB46 and MYB83 were considered to be a secondary master switch of SND1 involved in lignin synthesis [58, 60]. PtrMYB103/46 (Potri.003G132000), a homolog of AtMYB46, was also induced 
by high temperature although weakly. Some other MYB transcription factors such as PtrMYB002, PtrMYB003, PtrMYB020, PtrMYB161, PtrMYB90, PtrMYB152, PtrMYB189, etc. have also been reported to be involved in lignin synthesis [59, 60]. Transcriptome data showed that PtrMYB090 (Potri.015G033600), PtrMYB161 (Potri.007G134500), PtrMYB3 (Potri.001G267300), PtrMYB125/85 (Potri.003G114100), PtrMYB093 (Potri.004G138000) transcript expression levels were elevated by high temperature-induced expression. These PtrMYBs may play an important role in lignin accumulation of poplar stem in response to high temperature.

\section{Conclusions}

In general, when subjected to high temperature stress, poplar produces lignin through secondary metabolic pathways to resist adverse abiotic stresses. Several transcription factors and structural genes in lignin synthesis pathway participated in the high temperature response of poplar. In addition, the intermediates of lignin metabolic pathway (caffeate and coniferaldehyde) were also affected by high temperature and thus regulated lignin content. Identification of the key substances and genes in the lignin pathway in response to high temperature is of great significance to explore the high temperature resistance of poplar.

\section{Materials And Methods}

\section{Plant material and experimental conditions}

Poplar tissue culture plantlets "84k" were presented by Lingli Li of Northwest A\&F University and cultured under $25^{\circ} \mathrm{C}$ and $16 / 8$ hours long-day condition. Tissue culture plantlets for about 40 days old were placed in an incubator with $35^{\circ} \mathrm{C}$ treatment, and sampled at $0,6,12,24,48$, and 72 hours, respectively. The treatment time of $25^{\circ} \mathrm{C}$ was $0 \mathrm{~h}$ and $72 \mathrm{~h}$. The collected samples were quickly placed in liquid nitrogen and then stored in an ultra-cold refrigerator at $-80^{\circ} \mathrm{C}$.

\section{Determination of lignin content}

Lignin content was determined according to the kit description (Suzhou Comin Bioltechnology Co., Ltd, Suzhou, China). The sample was dried at $80^{\circ} \mathrm{C}$ to constant weight, crushed, passed through a 40-mesh sieve, and weighed out about $5 \mathrm{mg}$ (denoted as $\mathrm{W}$ ) in a $10 \mathrm{~mL}$ glass test tube. Add $1 \mathrm{~mL}$ bromoacetylglacial acetic acid and $40 \mu \mathrm{L}$ perchloric acid to the blank tube and sample tube, sealed the glass test tube with sealing film, mixed well, bathed in water at $80^{\circ} \mathrm{C}$ for 40 minutes, shaked every 10 minutes, then cooled naturally. Added $1 \mathrm{~mL} \mathrm{NaOH}$-acetic acid solution to glass test tube and mixed thoroughly. Took 40 $\mu \mathrm{L}$ supernatant and added $1960 \mu \mathrm{L}$ glacial acetic acid (adjusted the amount of glacial acetic acid properly to ensure the absorbance value between $0.1-0.8$ ) and mixed well. $1 \mathrm{~mL}$ of the mixed sample was placed in A quartz colorimetric dish to determine the absorbance value $A$ at $280 \mathrm{~nm}$. They were denoted as blank tube $A$ and measuring tube $A$ respectively. $\triangle A=A$ measuring tube $-A$ blank tube.

Lignin (mg/g dry weight) $=(\triangle \mathrm{A}-0.0068) / 0.0694 * \mathrm{~V} * 10^{-3} / \mathrm{W} * \mathrm{~T}$ 
V: total volume of reaction, $2.04 \mathrm{~mL}$; W: sample mass, g; T: dilution multiple

\section{Extraction and collection conditions of intermediate metabolites of lignin}

Measurement of intermediate metabolites of lignin was conducted by Metware Company with Ultra Performance Liquid Chromatography and Tandem Mass Spectrometry (UPLC-MS/MS). Samples were extracted as follows: biological samples were vacuum freeze-dried and ground ( $30 \mathrm{~Hz}, 1.5 \mathrm{~min})$ to powder form using a grinder (MM 400, Retsch). The $100 \mathrm{mg}$ of powder was weighed and dissolved in $0.6 \mathrm{~mL}$ of extraction solution, then the dissolved sample was placed in a refrigerator at $4^{\circ} \mathrm{C}$ for 12 hours. After centrifugation (10,000 $\mathrm{g}$ for $10 \mathrm{~min}$ ), the supernatant was aspirated and the sample was filtered through a microporous membrane $(0.22 \mu \mathrm{m}$ pore size $)$ and stored in the injection vial for UPLC-MS/MS analysis.

UPLC-MS/MS analysis contained liquid phase and mass spectrometry conditions. The specific steps and methods of the liquid phase were as follows: (1) Chromatographic column: Waters ACQUITY UPLC HSS T3 C18 $1.8 \mu \mathrm{m}, 2.1 \mathrm{~mm} * 100 \mathrm{~mm}$; (2) mobile phase: ultra-pure water (with $0.04 \%$ acetic acid) for phase A and acetonitrile (with $0.04 \%$ acetic acid) for phase B; (3) elution gradient: $5 \%$ for phase B at 0.00 min, linearly increasing to $95 \%$ for phase B at 10.00 min and maintaining at $95 \%$ for $1 \mathrm{~min}$, decreasing to $5 \%$ for phase $B$ from 11.00 to $11.10 \mathrm{~min}$, and balanced at $5 \%$ until $14 \mathrm{~min}$; (4) Flow rate $0.35 \mathrm{ml} / \mathrm{min}$; column temperature $40^{\circ} \mathrm{C}$; injection volume $4 \mu \mathrm{L}$. Mass spectrometry conditions were as follows: electrospray ionization (ESI) at $550^{\circ} \mathrm{C}$, mass spectrometry voltage $5500 \mathrm{~V}$, curtain gas (CUR) was 30 psi, collisionactivated dissociation (CAD) was high, declustering potential (DP) was optimized, and collision energy (CE) was scanned for detection [62].

The conditions and parameters of mass spectrometry were shown below: electrospray ionized (ESI) source temperature was $550^{\circ} \mathrm{C}$, CURTAIN gas was $30 \mathrm{PSI}$, mass spectrometry voltage was $5500 \mathrm{~V}$, along with high collisions activate dissociation, dissociation potential optimization, and collision energy scanning.

\section{Qualitative and quantitative analysis of metabolites}

Based on the self-built database MWDB (metware database) and the public database of metabolite information, qualitative analysis of primary and secondary spectra data from mass spectrometry assays was performed. The metabolite quantification was done by using multiple reaction monitoring (MRM) mode of triple quadrupole mass spectrometry. After obtaining the metabolite spectra of different samples, the peak areas of the mass spectra of all substances were integrated, and the integration of the mass spectra of the same metabolite in different samples was corrected [63].

The metabolites of the samples were analyzed qualitatively and quantitatively by mass spectrometry through the software analyst 1.6.3 which opens the browsing of the downstream raw data after the mass spectrometry analysis. The MRM metabolite detection multi-peak map showed the substances that could 
be detected in the samples, with each differently colored mass spectrometry peak representing one metabolite detected. The signal intensity (CPS) of the characteristic ions was obtained in the detector by screening each substance with a triple quadrupole, and the sample offline mass spectrometry file was opened with MultiaQuant software to perform peak integration and calibration work. A quality control sample was inserted in every 10 samples analyzed to monitor the reproducibility of the analysis process.

Fold Change (Fold Change) represented the ratio of expression between two samples (groups), and metabolites with fold change $\geq 2$ and fold change $\leq 0.5$ were selected as the final differential metabolites.

\section{Transcriptome sequencing}

Young poplar stems treated at different temperatures for different time periods were collected, rapidly frozen in liquid nitrogen, and later stored in an ultra-low temperature incubator at $-80^{\circ} \mathrm{C}$. Total RNA was extracted using a plant RNA extraction kit (Takara, Dalian, China) as described, while RNA concentration and purity were measured by NanoDrop 2000 (Thermo Fisher Scientific, Wilmington, DE, USA). RNA integrity was assessed using the RNA Nano 6000 assay kit from the Agilent Bioanalyzer 2100 system (Agilent Technologies, CA, USA). mRNA-seq library was created by mRNA-Seq Sample Preparation Kit (Cat \# RS-930-1001, Illumina Inc., San Diego, CA).

The RNA seq was performed on paired-end and data analysis was performed as follows [65]. The raw data was processed in fast format by an internal script. In this process, clean reads were obtained by removing reads containing adapters, reads containing ploy- $\mathrm{N}$, and low-quality reads of the raw data. At the same time, Q20, Q30, GC-content and sequence repeat levels of clean reads were calculated. These clean reads were then mapped to the reference genome sequence. On the basis of the reference genome, only the exact matches or mismatched reads were further analyzed and annotated. The reference genome was localized using Hisat2 software.

Gene expression levels were estimated by transcribed fragments per kilobase per million (FKPM) fragments. Differential expression analysis of the two samples was performed using R edge. The FDR < 0.01 \& Fold Change $\geq 2$ was used as the threshold for significant differences in expression. Gene functions were annotated based on the following databases: KOG/COG (Homology Protein Cluster); KO (KEGG Ortholog database); $\mathrm{Nr}$ (NCBI non-redundant protein sequences); Nt (NCBI non-redundant nucleotide sequences); (containing protein families); Swiss-Prot (a manually annotated and reviewed protein sequence database); (gene ontology). Gene Ontology (GO) enrichment analysis of differentially expressed genes (DEGs) was performed using $\mathrm{GO}$ seq $\mathrm{R}$ package based on the Wallenius non-central hypergeometric distribution [64]. KOBAS software was used to detect statistical enrichment of differentially expressed genes in KEGG pathway. P. trichocarpa V3.0 was used as the reference genome (https://phytozome.jgi.doe.gov/pz/portal.html).

\section{RT-qPCR (reverse transcription-quantitative real-time polymerase chain reaction) assays}

Page $12 / 24$ 
The transcript levels of PtrMYBs, PtrCCR2 and PtrC3'H1 were examined by RT-qPCR. PtrACTIN was used as the control. The execution was performed through SYBR Green Fluorescence method described in a previous article [66]. The cycling conditions for RT-qPCR were as follows: pre-denaturation at $95^{\circ} \mathrm{C}$ for 10 min, denaturation at $95^{\circ} \mathrm{C}$ for $10 \mathrm{~s}$, annealing at $60^{\circ} \mathrm{C}$ for $10 \mathrm{~s}$, and extension at $72^{\circ} \mathrm{C}$ for $30 \mathrm{~s}$ for a total of 40 cycles. Fluorescence signals were obtained th the extension phase of each cycle. Data analysis was performed by the 2- $\Delta \Delta \mathrm{Ct}$ method. The primers used in this experiment were shown in Table S1.

\section{Declarations}

\section{Acknowledgements}

We thank to Bioeditas Technology Corporation (Shanxi, China) for helping with transcriptome sequencing analysis and Metware Company for helping with targeted metabolome analysis.

\section{Authors' contributions}

T.X. and X.Z. designed the research project. X.Z., P.L., X.L., T.X., Y.Z. and H.M. performed the experiments and analyzed the data. X.Z. and T.X. wrote this manuscript. All authors read and approved the final manuscript.

\section{Funding}

This study was financially supported by the Integration of Science and Education Program Foundation for the Talents by Qilu University of Technology, Shandong Academy of Sciences (No. 2018-81110268) chaired by Tao Xia, Foundation of State Key Laboratory of Biobased Material and Green Papermaking (No. 2419010205, No. 23190444 chaired by Tao Xia and No. ZZ20200130 chaired by Xianyan Zhao).

\section{Availability of data and materials}

Data are available on request to the corresponding author. The transcriptome sequencing has been deposited under BioProjectin NCBI. The accession number for these SRA data is PRJNA742770.

\section{Ethics approval and consent to participate}

This study was carried out in State Key Laboratory of Biobased Material and Green Papermaking and School of Bioengineering of Qilu University of Technology in China and complies with relevant institutional, national, and international guidelines. " $84 k$ " (Populus albaxPopulus glandulosa) poplar tissue culture plantlets were used as experimental materials by kindly permission of Ling Lili of Northwest A\&F University. There is no ethics approval and consent to participate in this manuscript.

\section{Consent for publication}

Not applicable. 


\section{Competing interests}

The authors declare that they have no competing interests.

\section{Author details}

aState Key Laboratory of Biobased Material and Green Papermaking, School of Bioengineering, Qilu University of Technology, Jinan 250353, Shandong, PR China

${ }^{\mathrm{b}}$ Taian Forestry Protection and Development Center, Taian, 271018, Shandong, PR China

\section{References}

1. Nakahara Y, Tabata T, Ohno T, Furukawa F, Inokuchi K, Katagiri K, Hirayama Y. Discussion on regional revitalization using woody biomass resources as renewable energy. J Energ Eng.2019;10(2):243-256.

2. Zhao Q, Dixon RA. Transcriptional networks for lignin biosynthesis: more complex than we thought. Trends Plant Sci. 2011;16(4):227-233.

3. Van Acker R, Vanholme R, Storme V, Mortimer JC, Dupree P, Boerjan W. Lignin biosynthesis perturbations affect secondary cell wall composition and saccharification yield in Arabidopsis thaliana. Biotechnol Biofuels. 2013;6(1):46.

4. Behr M, Guerriero G, Grima-Pettenati, J, Baucher, M. A molecular blueprint of lignin repression. Trends Plant Sci. 2019;24(11):1052-1064.

5. Vanholme R, De Meester B, Ralph J, Boerjan W. Lignin biosynthesis and its integration into metabolism. Curr Opin Biotech. 2019;56:230-239.

6. Miao YC, Liu CJ. ATP-binding cassette-like transporters are involved in the transport of lignin precursors across plasma and vacuolar membranes. P Natl Acad Sci USA. 2010;107(52):2272822733.

7. Bonawitz ND, Chapple $C$. The genetics of lignin biosynthesis: connecting genotype to phenotype. Annu Rev Genet. 2010;44:337-363.

8. Vanholme R, Morreel K, Ralph J, Boerjan W. Lignin engineering. Curr Opin Plant Biol. 2008;11(3):278285.

9. Wang JP, Matthews ML, Williams CM, Shi R, Yang C, Tunlaya-Anukit S, Naik, P. Improving wood properties for wood utilization through multi-omics integration in lignin biosynthesis. Nature Commun. 2018;9(1):1-16.

10. Rohde A, Morreel K, Ralph J, Goeminne G, Hostyn V, De Rycke R, Van Driessche G. Molecular phenotyping of the pal1 and pal2 mutants of Arabidopsis thaliana reveals far-reaching consequences on phenylpropanoid, amino acid, and carbohydrate metabolism. Plant Cell. 2004;16(10):2749-2771. 
11. Chen F, Srinivasa Reddy MS, Temple S, Jackson L, Shadle G, Dixon RA. Multi-site genetic modulation of monolignol biosynthesis suggests new routes for formation of syringyl lignin and wall-bound ferulic acid in alfalfa (Medicago sativa L). Plant J. 2006;48(1):113-124.

12. Xie M, Zhang J, Tschaplinski TJ, Tuskan GA, Chen JG, Muchero W. Regulation of lignin biosynthesis and its role in growth-defense tradeoffs. Front Plant Sci. 2018; 9:1427.

13. Barros J, Serk H, Granlund I, Pesquet E. The cell biology of lignification in higher plants. Annals Of Botany. 2015;115(7):1053-1074.

14. Coleman HD, Park JY, Nair R, Chapple C, Mansfield SD. RNAi-mediated suppression of p-coumaroylCoA 3'-hydroxylase in hybrid poplar impacts lignin deposition and soluble secondary metabolism. P Natl Acad Sci USA. 2008;105(11):4501-4506.

15. Bryant ND, Pu Y, Tschaplinski TJ, Tuskan GA, Muchero W, Kalluri UC, Ragauskas AJ. Transgenic Poplar Designed for Biofuels. Trends Plant Sci. 2020;25(9):881-896.

16. Schoch G, Goepfert S, Morant M, Hehn A, Meyer D, Ullmann P, Werck-Reichhart D. CYP98A3 from Arabidopsis thaliana is a $3^{\prime}$-hydroxylase of phenolic esters, a missing link in the phenylpropanoid pathway. J Biol Chem. 2001;276(39):36566-36574.

17. Reddy MS, Chen F, Shadle G, Jackson L, Aljoe H, Dixon RA. Targeted down-regulation of cytochrome P450 enzymes for forage quality improvement in alfalfa (Medicago sativa $L$ ). Proceedings of the National Academy of Sciences. 2005;102(46):16573-16578.

18. Pu Y, Chen F, Ziebell A, Davison BH, Ragauskas AJ. NMR characterization of C3'H and HCT downregulated alfalfa lignin. BioEnerg Res. 2009;2(4):198.

19. Ralph J, Akiyama T, Coleman HD, Mansfield SD. Effects on lignin structure of coumarate 3hydroxylase down regulation in poplar. Bioenerg Res. 2012;5(4): 1009-1019.

20. Bonawitz ND, Im Kim J, Tobimatsu Y, Ciesielski PN, Anderson NA, Ximenes E, Chapple C. Disruption of Mediator rescues the stunted growth of a lignin-deficient Arabidopsis mutant. Nature. 2014; 509(7500): 376-380.

21. Ruel K, Berrio-Sierra J, Derikvand MM, Pollet B, Thévenin J, Lapierre C, Joseleau JP. Impact of CCR1 silencing on the assembly of lignified secondary walls in Arabidopsis thaliana. New Phytol. 2009;184(1):99-113.

22. Van Acker R, Leplé JC, Aerts D, Storme V, Goeminne G, Ivens B, Santoro N. Improved saccharification and ethanol yield from field-grown transgenic poplar deficient in cinnamoyl-CoA reductase. P Natl Acad Sci USA. 2014;111(2):845-850.

23. Tamasloukht B, Wong Quai Lam MS J, Martinez Y, Tozo K, Barbier O, Jourda C, Huguet, S. Characterization of a cinnamoyl-CoA reductase 1 (CCR1) mutant in maize: effects on lignification, fibre development, and global gene expression. J Exp Bot. 2011;62(11):3837-3848.

24. Leple JC, Dauwe R, Morreel K, Storme V, Lapierre C, Pollet B, Lefèbvre A. Downregulation of cinnamoyl-coenzyme A reductase in poplar: multiple-level phenotyping reveals effects on cell wall polymer metabolism and structure. Plant Cell. 2007;19(11):3669-3691. 
25. Ralph J, Kim H, Lu F, Grabber JH, Leplé J C, Berrio-Sierra J, Lapierre C. Identification of the structure and origin of a thioacidolysis marker compound for ferulic acid incorporation into angiosperm lignins (and an indicator for cinnamoyl CoA reductase deficiency). Plant J. 2008;53(2):368-379.

26. De Meester B, Calderón BM, de Vries L, Pollier J, Goeminne G, Van Doorsselaere J, Boerjan W. Tailoring poplar lignin without yield penalty by combining a null and haploinsufficient CINNAMOYLCOA REDUCTASE2 allele. Nat Commun. 2020; 11(1):1-13.

27. Kawasaki T, Koita H, Nakatsubo T, Hasegawa K, Wakabayashi K, Takahashi H, Shimamoto K. Cinnamoyl-CoA reductase, a key enzyme in lignin biosynthesis, is an effector of small GTPase Rac in defense signaling in rice. P Natl Acad Sci USA. 2006;103(1):230-235.

28. Daly P, McClellan C, Maluk M, Oakey H, Lapierre C, Waugh R, Halpin C. RNAi-suppression of barley caffeic acid O-methyltransferase modifies lignin despite redundancy in the gene family. Plant Biotechnol J. 2019;17(3):594-607.

29. Ni W, Paiva NL, Dixon RA. Reduced lignin in transgenic plants containing a caffeic acid Omethyltransferase antisense gene. Transgenic Res.1994;3(2):120-126.

30. Jouanin L, Goujon T, Nadaı V, Martin MT, Mila I, Vallet C, Pollet B, Yoshinaga A, Chabbert B, Petit-Conil $M$, Lapierre $C$. Lignification in transgenic poplars with extremely reduced caffeic acid Omethyltransferase activity. Plant Physiol. 2000;123 (4):1363-1374.

31. Tu Y, Rochfort S, Liu Z, Ran Y, Griffith M, Badenhorst P, Spangenberg G. Functional analyses of caffeic acid 0-methyltransferase and cinnamoyl-CoA-reductase genes from perennial ryegrass (Lolium perenne). Plant Cell. 2010;22(10): 3357-3373.

32. Saluja M, Zhu F, Yu H, Walia H, Sattler SE. Loss of COMT activity reduces lateral root formation and alters the response to water limitation in sorghum brown midrib (bmr) 12 mutant. New Phytol. 2021;229(5):2780-2794.

33. Ohtani M, Demura T. The quest for transcriptional hubs of lignin biosynthesis: Beyond the NAC-MYBgene regulatory network model. Curr Opin Biotech. 2019;56:82-87.

34. Lin YCJ, Chen H, Li Q, Li W, Wang JP, Shi R, Chiang VL. Reciprocal cross-regulation of VND and SND multigene TF families for wood formation in Populus trichocarpa. P Natl Acad Sci USA. 2017;114(45):E9722-E9729.

35. Taylor G. Populus: Arabidopsis for forestry. Do we need a model tree?. Annals Of Botany. 2002;90(6):681-689.

36. Moura JCMS, Bonine CAV, de Oliveira Fernandes Viana J, Dornelas MC, Mazzafera P. Abiotic and biotic stresses and changes in the lignin content and composition in plants. J Integr Plant Biol. 2010;52(4):360-376.

37. Zhu JK. Abiotic stress signaling and responses in plants. Cell.2016;167(2):313-324.

38. Cesarino I. Structural features and regulation of lignin deposited upon biotic and abiotic stresses. Curr Opin Biotechnol. 2019;56:209-214.

39. Crivellaro A, Büntgen U. New evidence of thermally constrained plant cell wall lignification. Trends Plant Sci. 2020;25(4):322-324. 
40. Zhao Q. Lignification: flexibility, biosynthesis and regulation. Trends Plant Sci. 2016;21(8):713-721.

41. Boerjan W, Ralph J, Baucher M. Lignin biosynthesis. Annu Rev Plant Biol. 2003;54(1):519-546.

42. Dixon RA, Paiva NL. Stress-induced phenylpropanoid metabolism. Plant Cell. 1995;7(7):1085.

43. Sgarbi E, Fornasiero RB, Lins AP, Bonatti PM. Phenol metabolism is differentially affected by ozone in two cell lines from grape (Vitis vinifera L.) leaf. Plant Sci. 2003;165(5):951-957.

44. Deng Y, Lu S. Biosynthesis and regulation of phenylpropanoids in plants. Crit Rev Plant Sci. 2017;36:257-290.

45. Reuter D. Nikki, Stewart C, Neal; Lenaghan, Scott C. Lighting the Way Advances in Engineering Autoluminescent Plants. Trends Plant Sci. 2020;25(12),1176-1179.

46. Bubna GA. Lima RB, Zanardo DYL, Santos WD, Ferrarese MLL, Ferrarese-Filho O. Exogenous caffeic acid inhibits the growth and enhances the lignification of the roots of soybean (Glycine max). J Plant Physiol. 2011;168(14):1627-1633.

47. Lapierre C, Pilate G, Pollet B, Mila I, Leplé J-C, Jouanin L, Kim H, Ralph J. Signatures of cinnamyl alcohol dehydrogenase deficiency in poplar lignins. Phytochemistry. 2004;65(3):313-321.

48. Anderson NA, Tobimatsu Y, Ciesielski PN, Ximenes E, Ralph J, Donohoe BS, Ladisch M, Chapple C. Manipulation of guaiacyl and syringyl monomer biosynthesis 1154 in an Arabidopsis cinnamyl alcohol dehydrogenase mutant results in atypical lignin biosynthesis and modified cell wall structure. Plant Cell. 2015;27:2195-2209.

49. Van Acker R, Déjardin A, Desmet S, Hoengenaert L, Vanholme R, Morreel K, Laurans F, Kim H, Santoro N, Foster C, Goeminne G, Légée F, Lapierre C, Pilate G, Ralph J, Boerjan W. Different routes for coniferand sinapaldehyde and higher saccharification upon deficiency in the dehydrogenase CAD1. Plant Physiol. 2017; 175(3):1018-1039.

50. Grabber JH, Ralph J, Hatfield RD. Severe inhibition of maize wall degradation by synthetic lignins formed with coniferaldehyde. J Sci Food Agr.1998;78(1):81-87.

51. Fornalé S, Capellades M, Encina A, Wang K, Irar S, Lapierre C, Ruel K, Joseleau J, Berenguer J, Puigdomènech P, Rigau J, Caparrós-Ruiz D. Altered lignin biosynthesis improves cellulosic bioethanol production in transgenic maize plants down-regulated for cinnamyl alcohol dehydrogenase. Mol Plant. 2012;5(4): 817-830.

52. Blaschek L, Nuoendagula, Bacsik Z, Kajita S, Pesquet E. Determining the genetic regulation and coordination of lignification in stem tissues of arabidopsis using semiquantitative raman microspectroscopy. ACS Sustain Chem Eng. 2020;8(12):4900-4909.

53. Mottiar Y, Vanholme R, Boerjan W, Ralph J, Mansfield SD. Designer lignins: harnessing the plasticity of lignification. Curr Opin Biotechnol. 2016;37:190-200.

54. Abdulrazzak N, Pollet B, Ehlting J, Larsen K, Asnaghi C, Ronseau S, Proux C, Erhardt M, Seltzer V, Renou JP, Ullmann P, Pauly M, Lapierre C Werck-Reichhart D. A coumaroyl-ester-3-hydroxylase insertion mutant reveals the existence of nonredundant meta-hydroxylation pathways and essential roles for phenolic precursors in cell expansion and plant growth. Plant Physiol. 2006;140(1):30-48. 
55. Allina SM, Pri-Hadash A, Theilmann DA, Ellis BE, Douglas CJ. 4-Coumarate: coenzyme A ligase in hybrid poplar: properties of native enzymes, cDNA cloning, and analysis of recombinant enzymes. Plant Physiol. 1998;116(2):743-754.

56. Hu WJ, Kawaoka A, Tsai CJ, Lung J, Osakabe K, Ebinuma H, Chiang VL. Compartmentalized expression of two structurally and functionally distinct 4-coumarate: CoA ligase genes in aspen (Populus tremuloides). Proc Natl Acad Sci USA. 1998;95(9):5407-5412.

57. Naik P, Wang JP, Sederoff R, Chiang V, Williams C, Ducoste JJ. Assessing the impact of the 4CL enzyme complex on the robustness of monolignol biosynthesis using metabolic pathway analysis. PLoS One. 2018;13(3):e0193896.

58. Zhong R, Richardson EA, Ye ZH. The MYB46 transcription factor is a direct target of SND1 and regulates secondary wall biosynthesis in Arabidopsis. Plant Cell. 2007;19(9):2776-2792.

59. Chen H, Wang JP, Liu H, Li H, Lin YCJ, Shi R, Sederoff RR. Hierarchical transcription factor and chromatin binding network for wood formation in Populus trichocarpa. Plant Cell. 2019;31(3):602626.

60. Ko JH, Jeon HW, Kim WC, Kim JY, Han KH. The MYB46/MYB83-mediated transcriptional regulatory programme is a gatekeeper of secondary wall biosynthesis. Annals Of Botany. 2014;114(6):10991107.

61. Wang Z, Mao Y, Guo Y, Gao J, Liu X, Li S, ... \& Li, W. MYB transcription factor161 mediates feedback regulation of secondary wall-associated NAC-Domain1 family genes for wood formation. Plant Physiol. 2020;184(3):1389-1406.

62. Chen W, Gong L, Guo Z, Wang W, Zhang H, Liu X, Yu S, Xiong L. A Novel Integrated Method for LargeScale Detection, Identification, and Quantification of Widely Targeted Metabolites: Application in the Study of Rice Metabolomics[J]. Mol Plant. 2013;6(6):1769-1780.

63. Fraga CG, Clowers BH, Moore RJ, Zink EM. Signature-discovery approach for sample matching of a nerve-agent precursor using liquid chromatography-mass spectrometry, XCMS, and chemometrics. Anal Chem. 2010; 82(10): 4165-73.

64. Young MD, Wakefield MJ, Smyth GK, Oshlack A. Gene ontology analysis for RNA-seq: accounting for selection bias. Genome Biol. 2010;11(2):1-12.

65. Jiang C. Efficient extraction of RNA from various Camellia species rich in secondary metabolites for deep transcriptome sequencing and gene expression analysis. Afr J Biotechnol. 2019; 10(74):1676316768.

66. Zhao XY, Qi CH, Jiang H, Zhong MS, You CX, Li YY, Hao YJ. MdWRKY15 improves resistance of apple to Botryosphaeria dothidea via the salicylic acid-mediated pathway by directly binding the MdICS1 promoter. J Integr Plant Biol. 2020:62(4):527-543.

\section{Figures}




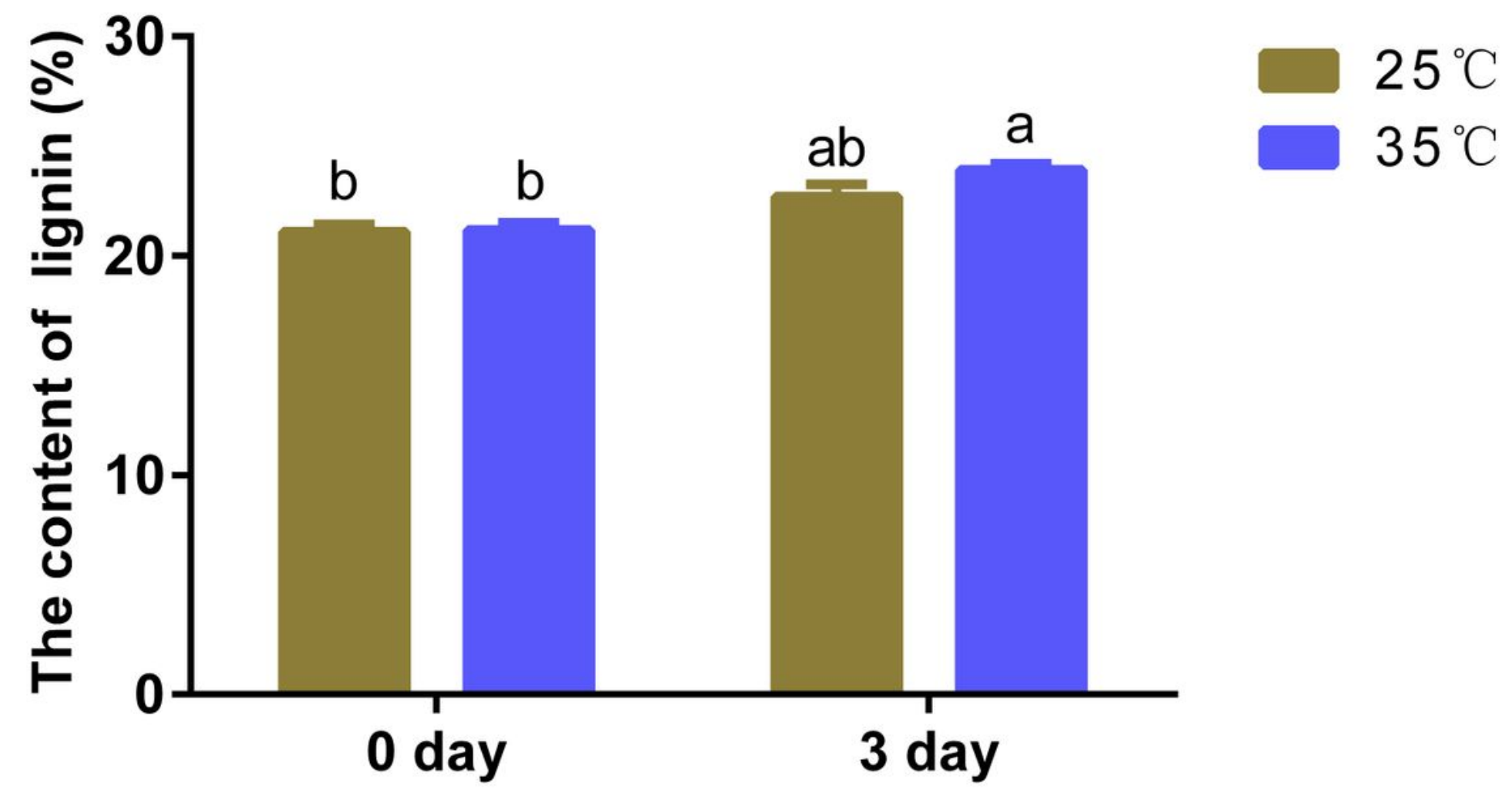

Figure 1

High temperature changed the lignin content in poplar. Lignin content of poplar stem after treatment of $35^{\circ} \mathrm{C}$ (heat treatment) and $25^{\circ} \mathrm{C}$ (normal treatment). The same letter of P0.05 level represents the no difference while different P0.05 level showed the imparity. The data showed as the mean \pm SE was repeated three times. $a$ and $b$ represent significant differences, while $a b$ and $a$ or $a b$ and $b$ are not significantly different in both pair because they have duplicate letters. 
Ferulic acid

Sinapinaldehyde

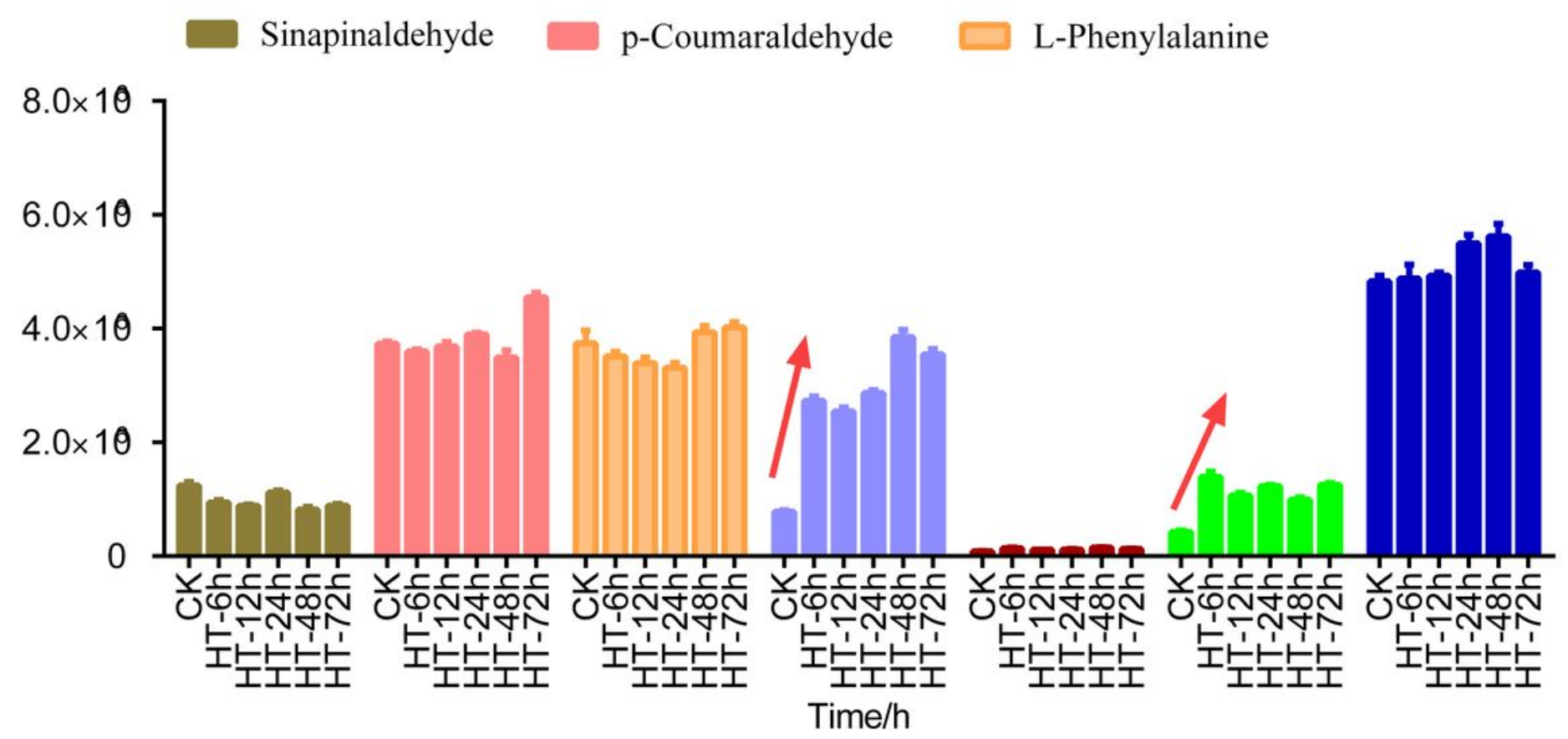

coniferaldehyde

p-Coumaraldehyde
Cinnamic acid

L-Phenylalanine

Figure 2

Analysis of intermediates in lignin pathway. Histogram of lignin level of 0 (CK), 6, 12, 24, 48, 72 hours after treated with cold stimulus. The data showed as the mean \pm SE was repeated three times. HT means high temperature. 

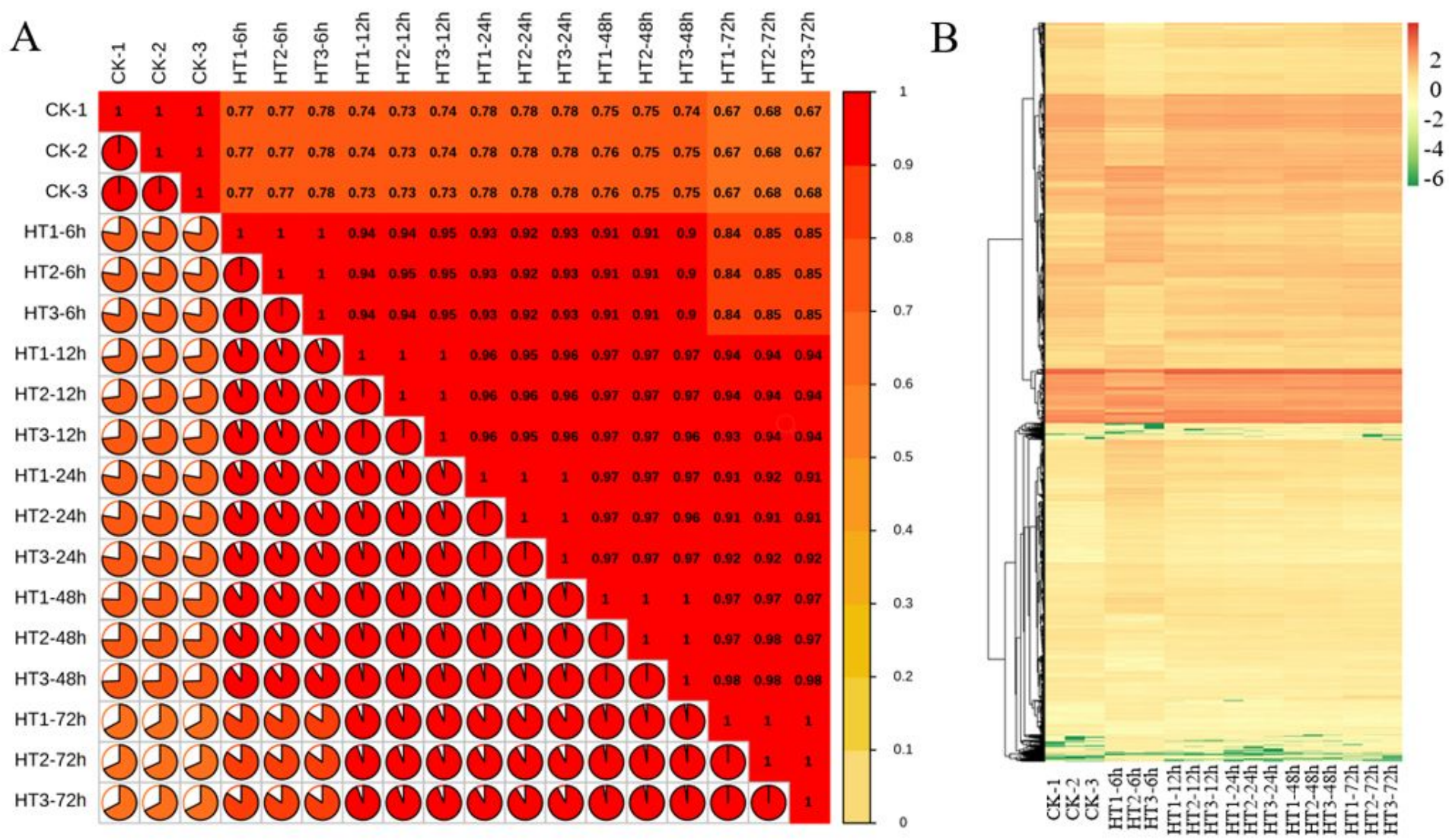

Figure 3

Analysis of gene expression in response to high temperature. A: Heat map of correlation coefficient values across samples based on RNAseq FPKM. B: Cluster analysis of DEGs of 0 (CK), 6, 12, 24, 48, 72 hours after treated with high temperature treatment. CK is the sample of 0 hour. HT means high temperature. DEG means differential expressed genes.

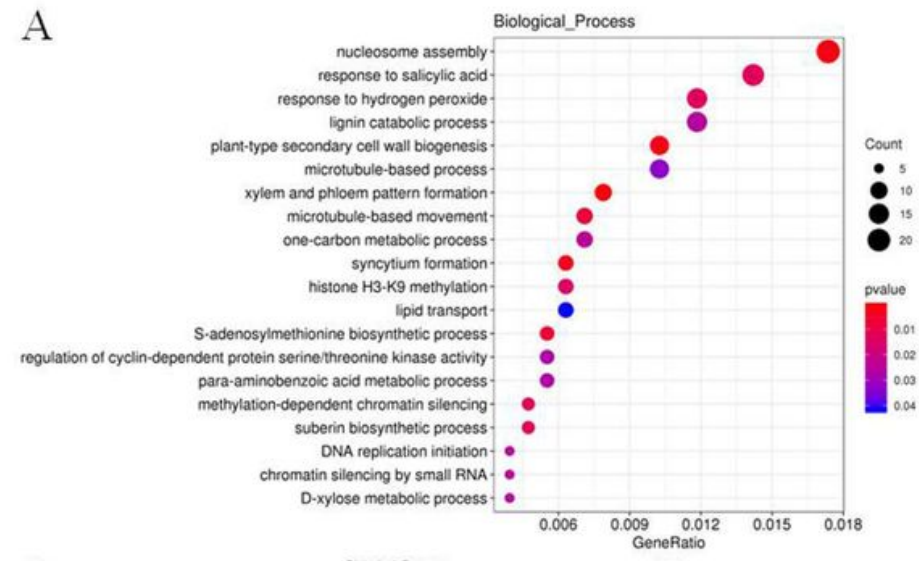

$\mathrm{C}$

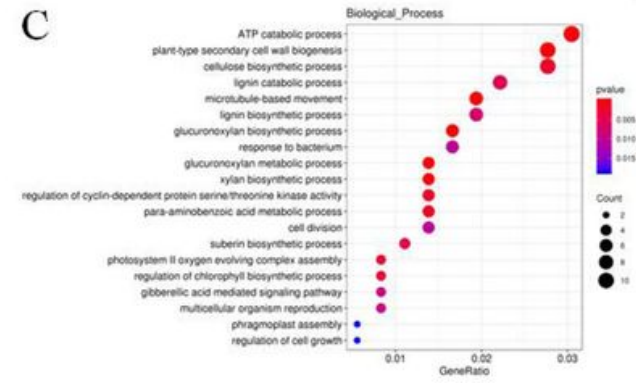

$\mathrm{D}$
B

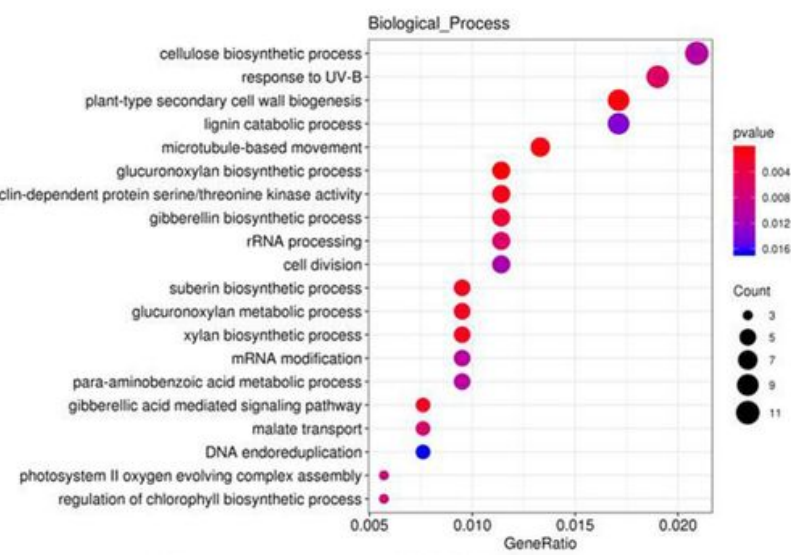

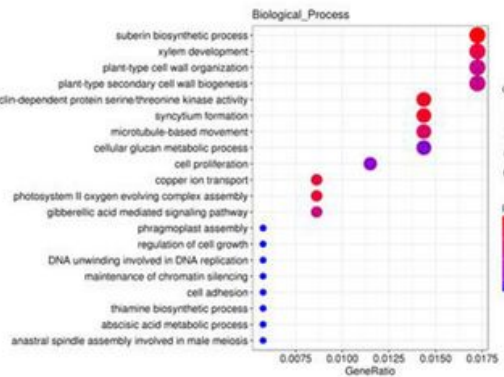
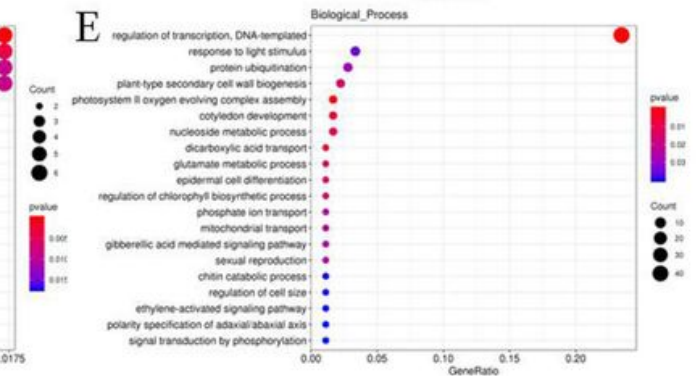

Page $21 / 24$ 


\section{Figure 4}

GO enrichment analysis of DEG. CK is the sample of 0 hour. HT means high temperature. DEG means differential expressed genes.

A

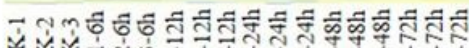
ช。ำ
4 B 6 h VS Oh $12 \mathrm{~h}$ VS ohl $24 \mathrm{~h}$ VS Oh $48 \mathrm{~h}$ VS 0h $72 \mathrm{~h}$ VS oh

$1.88662841 .84963346 \quad 1.75723135 \quad 1.22049213 \quad 1.12809002$ PtrC3'H1

$\begin{array}{llllll}1.71986274 & 0.89769883 & 0.87347598 & 0.99818329 & 1.15950747 & \text { PtrCCR2 }\end{array}$

\begin{tabular}{l|l|l|l|l|l|l|l}
5.31119351 & 3.32591467 & 2.75277052 & 1.71290192 & 2.10801729 & PtrMYB021
\end{tabular}

\begin{tabular}{l|lllll}
4.65619265 & 2.4757742 & 2.16489636 & 1.61189035 & 1.55801929 & PtrMYB074
\end{tabular}

$\begin{array}{lllllll}3.29823456 & 1.99132406 & 2.18783644 & 1.31806147 & 1.23733341 & \text { PtrMYB103/46 }\end{array}$

$\begin{array}{lllllll}2.5203436 & 4.75147341 & 3.31222335 & 1.98262648 & 1.37949764 & \text { PtrMYB090 }\end{array}$

$\begin{array}{llllll}1.96226622 & 2.46636586 & 1.75445328 & 1.81856522 & 1.30823091 & \text { PtrMYB161 }\end{array}$

\begin{tabular}{ll|l|l|l|l|l}
7.50864848 & 4.39762681 & 3.10138915 & 2.56882005 & 2.1021985 & PtrMYB3
\end{tabular}

$\begin{array}{lllllll}0.95416643 & 1.94883004 & 2.21979257 & 1.17909624 & 1.27574238 & \text { PtrMYB125/85 }\end{array}$

$\begin{array}{llllll}2.17728732 & 1.17931199 & 1.09776008 & 1.71240092 & 1.18070248 & \text { PtrMYB093 }\end{array}$

\section{Figure 5}

The variation trend of lignin-related genes induced by heat stress. A: Cluster analysis of lignin-related genes induced by heat stress. $B$ : The expression level of some lignin-related genes at different time periods $(6,12,24,48,72$ hours) compared with the control group ( 0 hour) after HT treatment. CK is the sample of 0 hour. HT means high temperature. DEG means differential expressed genes.

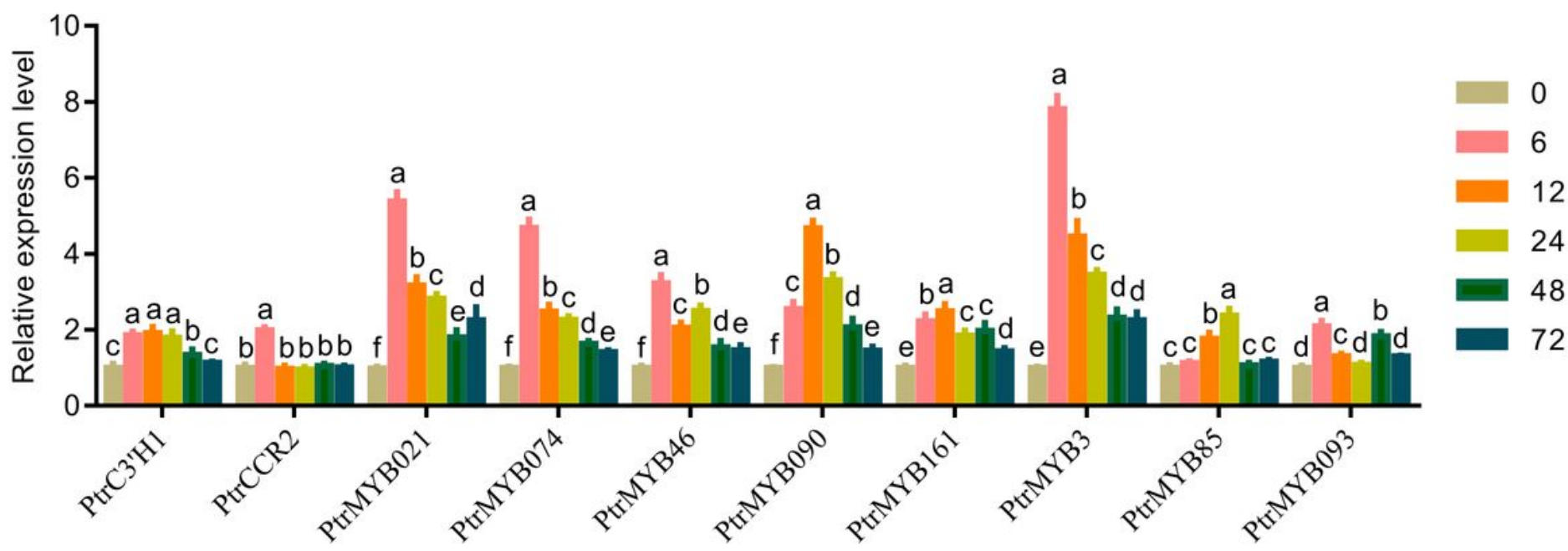

Figure 6

RT-qPCR analysis of lignin-related genes. RT-qPCR analysis of PtrC3'H1 (Potri.006G033300), PtrCCR2 (Potri.003G181400), PtrMYB021 (Potri.009G053900) and PtrMYB074 (Potri.015G082700) \ PtrMYB103/46 (Potri.003G132000), PtrMYB090 (Potri.015G033600), PtrMYB161 (Potri.007G134500), PtrMYB3 (Potri.001G267300), PtrMYB125/85 (Potri.003G114100), PtrMYB093 (Potri.004G138000) of 
poplar stem with high temperature treatment for $0,6,12,24,48$ and 72 hours. The same letter of P0.05 level represents the no difference while different P0.05 level showed the imparity. The data showed as the mean $\pm S E$ was repeated three times. between

\section{Transcriptome analysis}
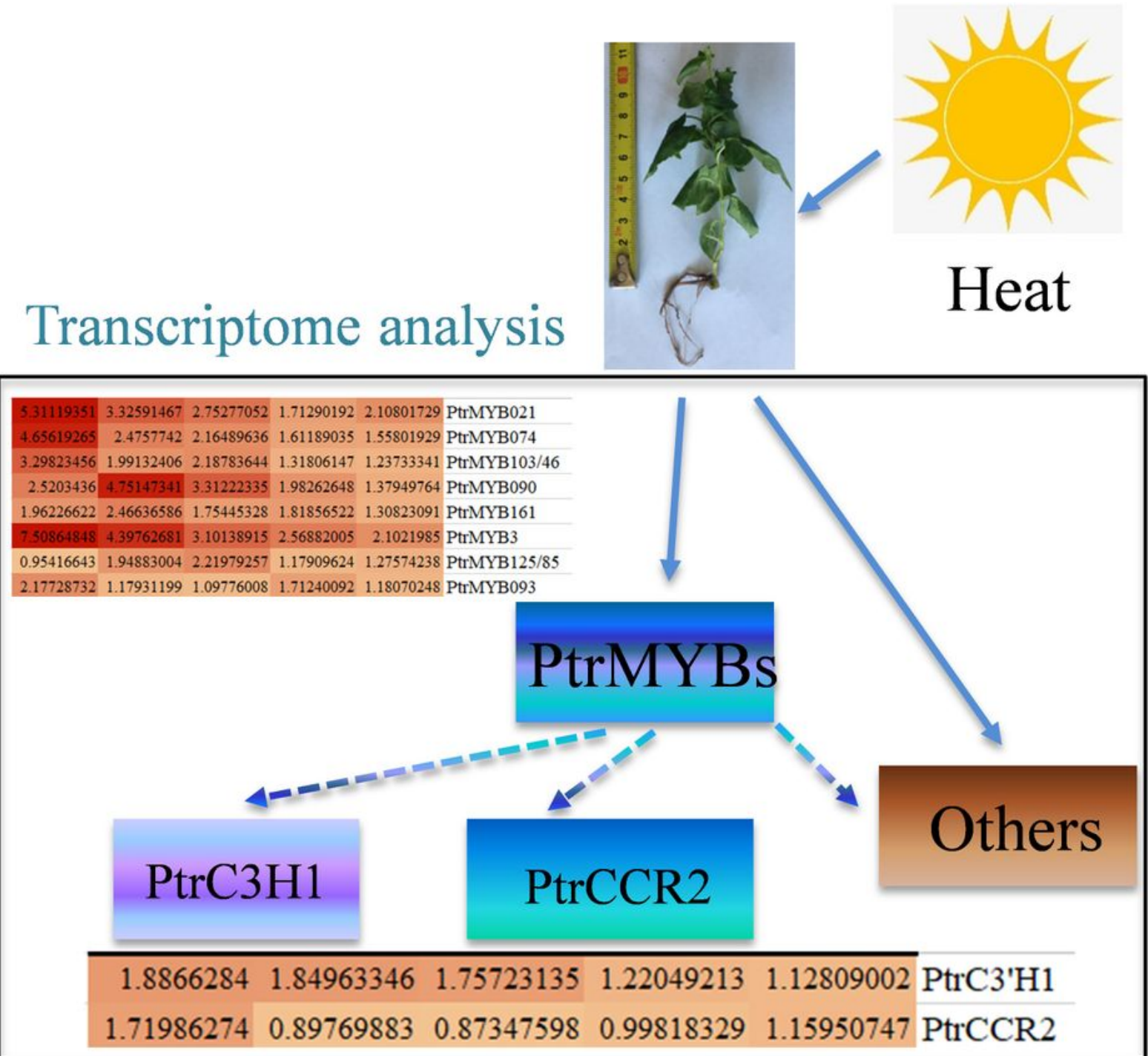

Metabolite analysis $\downarrow$

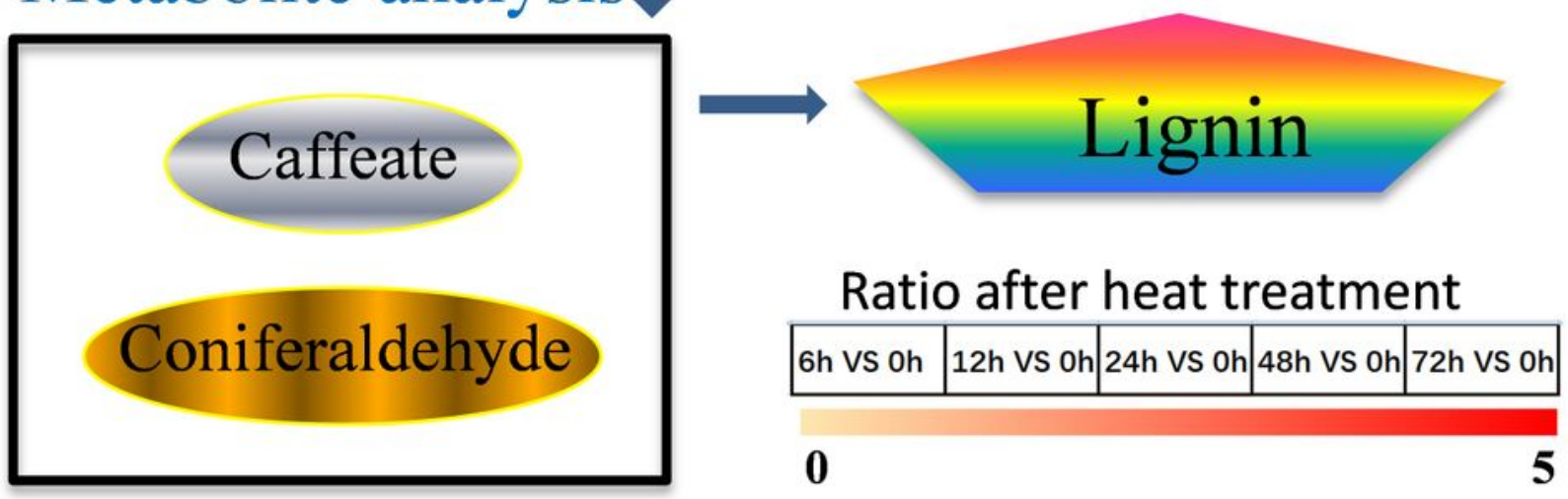

Figure 7

Regulatory mechanism between high temperature and lignin content. High temperature brings about the increased lignin content. Transcriptome analysis indicated that some vital transcription factors such as 
PtrMYBs, others lignin related transcription factors and lignin-related structural gene such as $\mathrm{PtrC}^{\prime} \mathrm{H} 1$, PtrCCR2 were induced by high temperature. PtrC3'H1 may be a possible factor leading to the decrease of caffeate content, while PtrCCR2 may bring about the lessen of coniferaldehyde. caffeate, coniferaldehyde represents the different lignin precursors.

\section{Supplementary Files}

This is a list of supplementary files associated with this preprint. Click to download.

- SupplementalTable1.doc

- Supplementarymaterials1.xlsx

- Supplementarymaterials2.xls

- Supplementarymaterials4.xlsx

- Supplementarymaterials3.xlsx

- Supplementarymaterials5.xlsx

- Supplementarymaterials6.xlsx

- Supplementarymaterials7.xIsx

- Supplementarymaterials8.xIsx 